\title{
Impaired Right Coronary Vasodilator Function in Pulmonary Hypertensive Rats Assessed by In Vivo Synchrotron Microangiography
}

\author{
Tadakatsu Inagaki, Hirotsugu Tsuchimochi, \\ James T. Pearson, Daryl O. Schwenke, Keiji Umetani, \\ Mikiyasu Shirai, and Yoshikazu Nakaoka
}

\section{Keywords}

Coronary microangiography - Synchrotron radiation · Right heart failure • Endothelial dysfunction

Pulmonary hypertension (PH) causes cardiac hypertrophy in the right ventricle (RV) and eventually leads to RV failure due to persistently elevated afterload. Considerable clinical and experimental animal studies have shown that abnormalities of endothelial function represent a hallmark of heart failure. However, the relationships between right coronary endothelial dysfunction and the development of heart failure remain to be fully established especially in the right heart. Synchrotron

\footnotetext{
T. Inagaki $\cdot$ Y. Nakaoka $(\bowtie)$

Department of Vascular Physiology, National Cerebral and Cardiovascular Center,

Osaka, Japan

e-mail: ynakaoka@ncvc.go.jp

H. Tsuchimochi · J. T. Pearson

Department of Cardiac Physiology, National Cerebral and Cardiovascular Center,

Osaka, Japan

D. O. Schwenke

Department of Physiology, University of Otago, Dunedin, New Zealand

K. Umetani

Japan Synchrotron Radiation Research Institute, Sayo-gun, Hyogo, Japan

M. Shirai

Department of Advanced Medical Research for Pulmonary Hypertension, National Cerebral and Cardiovascular Center, Osaka, Japan
} 
radiation (SR) microangiography has provided the temporal and spatial resolution required to visualize microvessels of various organs in vivo [1]. Therefore, in this study, we aimed to validate a new approach for the in vivo assessment of endothelial coronary function of the rat right heart using SR microangiography.

Imaging of the right coronary circulation was performed under pentobarbital anesthesia with monochromatic SR at $33.2 \mathrm{keV}$ for producing maximal absorption contrast of the iodine contrast agent in the vascular lumen (Fig. 25.1a). ImageJ (ver. 1.41, NIH, Bethesda, MD) was used to identify coronary vessels and determine their caliber. Vessels were labeled and classified according to branching orders from first-order main segment (Fig. 25.1b). The visible vessel internal diameter (ID) of each vessel was determined from a single field of view for all cine sequences. The ID of each vessel was averaged over at least ten consecutive frames [2].

The figure shows a typical imaging pattern of right coronary arteries from the aortic root to the third or fourth branching. It was possible to observe an arteriole with a diameter of about $50 \mu \mathrm{m}$ (Fig. 25.1c). The ID of the first, second, and third

a

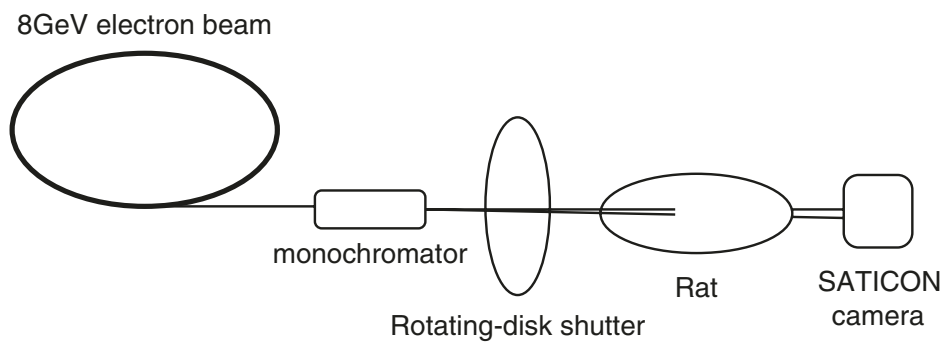

b

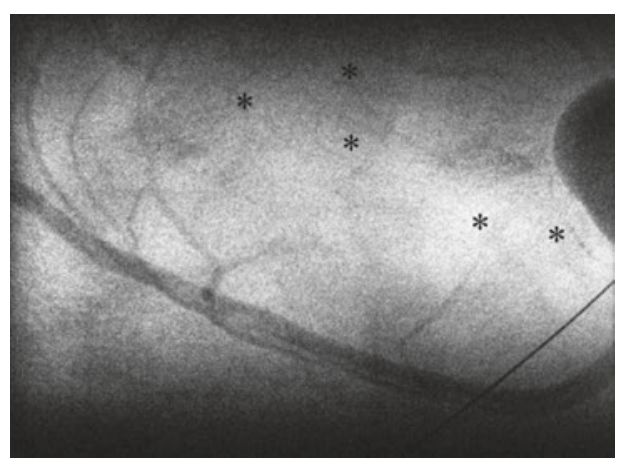

\section{C}

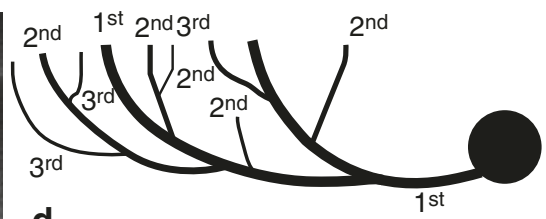

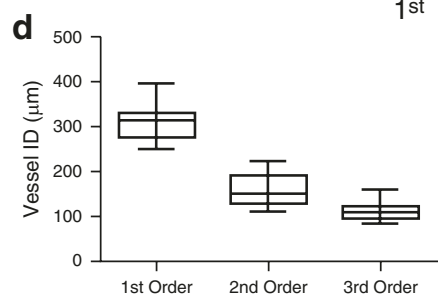

Fig. 25.1 SR microangiography for assessing vascular function in a rat. Schematic of SR microangiography for the rat right coronary artery using SPring-8 facilities (a). Typical SR angiogram image of the right coronary vasculature (b). Schematic of right coronary arteries in lateral view depicting the coronary branching nomenclature used in this study (c). Range of vessel size at each of the branching generations of right coronary circulation in the normal rat (d). Asterisks indicate $\sim 50 \mu \mathrm{m}$ vessels not included in (d) 
branches were $312.7 \pm 15.4,159.3 \pm 12.4$, and $113.1 \pm 7.5 \mu \mathrm{m}$, respectively (Fig. 25.1d). The present investigation demonstrates the ability to clearly visualize the right coronary circulation of the closed-chest anesthetized rat using SR microangiography. The use of SR microangiography provides a powerful tool for assessing coronary hemodynamics in unprecedented detail in PH models. Ultimately, future studies using SR microangiography will provide important new insights into the pathophysiology of right heart failure.

Acknowledgments Experiments were performed at the Japan Synchrotron Radiation Research Institute (SPrimg-8, BL28B2, Proposals 2014B1801 and 2015A1868). This work was supported by JSPS KAKENHI JP25860184 to T.I.

\section{References}

1. Shirai M, et al. Synchrotron radiation imaging for advancing our understanding of cardiovascular function. Circ Res. 2013;112:209-21.

2. Jenkins MJ, et al. Dynamic synchrotron imaging of diabetic rat coronary microcirculation in vivo. Arterioscler Thromb Vasc Biol. 2012;32:370-7.

Open Access This chapter is licensed under the terms of the Creative Commons Attribution 4.0 International License (http://creativecommons.org/licenses/by/4.0/), which permits use, sharing, adaptation, distribution and reproduction in any medium or format, as long as you give appropriate credit to the original author(s) and the source, provide a link to the Creative Commons license and indicate if changes were made.

The images or other third party material in this chapter are included in the chapter's Creative Commons license, unless indicated otherwise in a credit line to the material. If material is not included in the chapter's Creative Commons license and your intended use is not permitted by statutory regulation or exceeds the permitted use, you will need to obtain permission directly from the copyright holder.

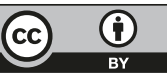

